\title{
Multi-Band Spectrum Allocation Algorithm Based on First-Price Sealed Auction
}

\author{
Zijuan Shi ${ }^{1}$, Gaofeng Luo $^{2}$ \\ ${ }^{1}$ Department of Foreign Languages, Shaoyang University, Shaoyang, China \\ ${ }^{2}$ Key Laboratory of Information Service for Rural Area of Southwestern Hunan, Shaoyang University, \\ Shaoyang, China \\ Emails: zijuanshi@126.com gfluo@126.com
}

Abstract: Auction is often applied in cognitive wireless networks due to its fairness properties and efficiency. To solve the allocation issues of cognitive wireless network in a multi-band spectrum, multi-item auction mechanism and models were discussed in depth. Multi-item highest price sealed auction was designed for cognitive wireless networks' multi-band spectrum allocation algorithm. This algorithm divided the spectrum allocation process into several stages which was along with low complexity. Experiments show that the algorithm improves the utilization of spectrum frequency, because it takes into account the spectrum owner's economic efficiency and the users' equity.

Keywords: Cognitive radio networks, spectrum allocation, auction, secondary user.

\section{Introduction}

With the issue of $4 \mathrm{G}$ licenses in many countries, competition for wireless spectrum between operators increases in recent years making spectrum resources more valuable. The measured results of spectrum utilization conducted in University of California, Berkeley [1] show that, in the United States and Europe, only about 10\% of all the allocated spectrum resources are fully utilized. The usage of some licensed bands is at a low level. Some new wireless applications are concentrated in the unlicensed bands, which are overcrowded.

To realize efficient spectrum usage, Cognitive Radio (CR) [2] technology came into being. Its basic function is to integrate spare spectrum, providing users with access to services to choose spectrum [3,4]. Currently, Wireless Local Area Network (WLAN) is widely deployed in airports, hotels and other places, which makes cognitive radio highly needed. CR terminals can rent short-term spectrum according to demands, then Internet Service Provider transfers out the user data. Similarly, with the CR module embedded in smart phones, these smart phones can also connect with spare WLAN even without the operator's license $[5,6]$. Thus the spectrum is efficiently utilized, realizing wireless communications. 
The remainder of this work is structured as follows. Section 2 reviews some related works. Then we introduce the proposed multi-band spectrum allocation algorithm in Section 3. Experimental results are reported in Section 4 with rigorous analysis. Finally, the concluding remarks are provided in Section 5.

\section{Related works}

The first research on distribution of the spectrum was suggested for the spectrum pool system proposed by F. K. Jondral professor at the Karlsruhe University. The system consists of a CR base station and a plurality of mobile radio users, providing a network structure for cognitive radio spectrum allocation [3]. The base station completed the detection of the authorized users by periodically broadcasting detection frame, and at the same time gathers sensory information. The basic idea of spectrum pool was to take channel as the basic unit of spectrum allocation, and to maximize the channel utilization, taking into account the interference among systems.

Recently, the application of auction theory to spectrum allocation has been a major concern of many researchers [4, 5, 6]. Gandhi et al. [7] proposed a low complexity framework to achieve real-time dynamic spectrum auctions, using two price models to weigh the benefits and fairness of the system. Chen, Ho ang and Liang [8], and Bin Chen et al. [9] first proposed an auction mechanism in which cognitive users can get access to channel via time slots during competitive bidding. In addition, the author also proposed an optimization strategy based on the second-price auction cognitive radio spectrum allocation. Ni y a to and $\mathrm{Hos}$ sian [10] analyzed the problem of multiple authorized users facing a single cognitive user spare spectrum, he believed that through the multi-stage repeated bidding it had the potential to bring more serious conspiracy. Performance evaluation of the proposed scheme shows the ability of the scheme to maximize the reported revenue for the primary users under different spectrum market conditions [11].

In short, since the concept of cognitive radio was put forward, researches on $\mathrm{CR}$ and spectrum allocation have never ceased. It is expected to solve the problems of lacking for spectrum resources, and provide a bright prospect for wireless application.

\section{Our methods}

\subsection{System assumption}

By analyzing dynamic spectrum allocation for cognitive radio network, and considering the actual situation, we designed a model with a plurality of Spectrum Owner (referred to as SO), and a number of Secondary Users (referred to as SU) in the network. It should be noted that in many documents several spectrum owners are directly combined to one single spectrum broker (or spectrum server) which is in charge of spectral allocation in all spare channels. However, by the above analysis we can see that it is difficult for a broker to resolve all the problems of spectrum in the real network. 
First, there may not be a public control channel to exchange information between various owners of spectrum. Second, even if there is such a channel, it is hard to realize communication between the owners of the spectrum due to different signaling formats. Finally, if signaling interaction is realizable, it may cause too much communication spending.

Therefore, this article will propose an algorithm based on network structure and model. As shown in Fig. 1, the owners of spectrum in the system are SO1 and $\mathrm{SO} 2$, and each SO has several idle channels. Assuming the same SO is with the same channel bandwidth and modulation methods, and there are several SUs in the system. For each cognitive user $i(i=1,2, \ldots, N)$, the packets reach to buffer area at random, and the packet arrival rate is assumed to obey the mathematical expectation $\lambda$ of the Poisson distribution. Here we assume that the transmitter is fixed and transmits signals to a receiver via a wireless fading channel (channel correlation coefficients do not change in an auction cycle); the receiver will not move in the auction period or at a very slow speed mobile. Each cognitive user can determine whether bid or not according to the cognitive channel capacity and their own buffer groups, and bid to which spectrum owner, then officially launch the bid according to its own estimate and the budget. Finally, we also assume that each cognitive user can launch a bid to only one SO at the same time, and can only obtain one spare channel in an auction.

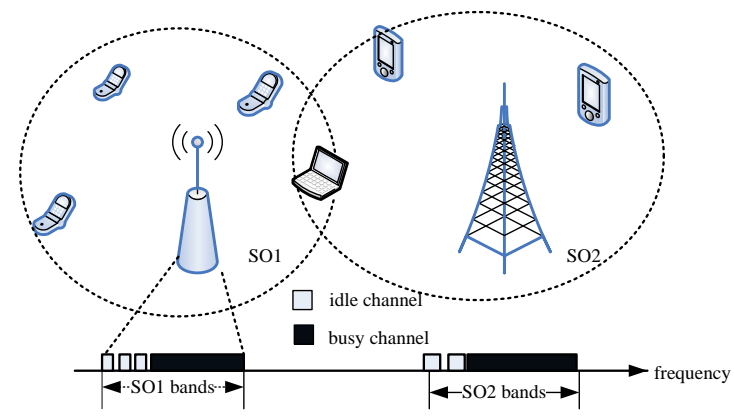

Fig. 1. CR networks model

\subsection{System model}

In the study of CR spectrum allocation based on auction theory, we assume that there is a cognitive radio network with $\mathrm{SO}(=M)$ and $\mathrm{SU}(=N)$. For spectrum owner $j$ $(j=1,2, \ldots, M)$, there might be $m_{j}$ temporary available channels and the spectrum owner holds an initial reserve price $r_{j}$. It is assumed that there are $k(k \leq N)$ cognitive users interested to the spare channels.

Each cognitive user $i(i=1,2, \ldots, N)$ has a valuation $v_{i j}$ for each spectrum owners $j$ before bidding for the channel, the valuation can be defined as the benefits of cognitive users, which is determined by the Shannon capacity of the channel itself and the number of groups within the cognitive user buffer. In order to estimate the channel status, it may be assumed that for each cognitive user, its packet arrival rate is a fixed $\lambda$, where $W_{j}$ is the Shannon capacity of the channel spectrum owned by the owner of $j$, namely 


$$
W_{j}=\log _{2}\left(1+\frac{h p}{N_{0}}\right),
$$

wherein $h$ is the channel correlation coefficient, $N_{0}$ is the average noise power, and $p$ is the signal transmission power. Thus we obtained:

$$
v_{i j}=1-\frac{\lambda}{\log _{2}\left(1+\frac{h p}{N_{0}}\right)} .
$$

$V=\left\{v_{i j}\right\}_{N \times M}$ defined as the channel valuation matrix of all SO and SU. Since each spare channel can only be assigned to one cognitive user, each cognitive user at a time can use only one spare channel. $C=\left\{c_{i j}\right\}_{N \times M}$ is defined as the assigned matrix, where $c_{i j}=0$ indicates a spectrum owner $i$ does not assign spare channel to cognitive user $i, c_{i j}=1$ represents a spare channel is allocated to cognitive user $i$. Therefore, the allocation matrix should be met

$$
\begin{gathered}
\sum_{i=1}^{N} c_{i j} \leq m_{j}, j=1,2, \ldots, M, \\
\sum_{j=1}^{M} c_{i j} \leq 1, i=1,2, \ldots, N,
\end{gathered}
$$

wherein (3) shows that each SO can only assign $m_{j}$ spare channel to cognitive users in order to avoid interference, and formula (4) indicates that each SU can only get one channel auction.

From the above analysis it can be concluded that the spectral efficiency of spectral owner $j$ to cognitive user $i$ is $v_{i j}-r_{j}$, if $r_{j}$ is 0 , then the overall system spectral efficiency is the total throughput of the system. Therefore, the spectral efficiency maximum can be obtained by

$$
R^{*}=\arg \max _{R} \sum_{i=1}^{N} \sum_{j=1}^{M} c_{i j}\left(v_{i j}-r_{j}\right) .
$$

For (5), it must satisfy the conditions of (3) and (4), and $c_{i j}$ can only get a value between 0 and 1 for any $i$ and $j$. In theory, the above problem is a NP-hard problem. In the CR network system, the entire valuation matrix information can not be obtained, plus the elusive information exchange between different spectrum owners, so that part of the value of the estimate matrix $V$ is also not available.

\section{Auction algorithm design and performance analysis}

\subsection{Algorithm design}

We divided the whole auction process into two stages: in the first stage, if a cognitive user $i$ had data packet to be transmitted, after scanning spectrum information around them, it transmitted a " 1 " bits to the owner of the spectrum, then the spectrum owner dynamically worked out a reserve price $r_{j}$ according to the number of spare channels and the number of current cognitive users who are interested in participating in the tender; in the second stage, cognitive user $i$ started to bid according to the information of the previous stage and the number of buffer packets, the bid value is bi, the spectral owner sorted all the bid values, and 
allocated its spare channels to the cognitive users who offer the highest price.

In the first stage, the spectrum owners need to count the number of cognitive users. We set the number of cognitive owners as $n_{j}$, which is involved in the bid for spare channel of spectrum owner $j$. If $n_{j} \leq m_{j}$, it indicates supply exceeding demand. At this time, $r_{j}$ can be defined as power consumption or interference from authorized user when the spectrum owner assigns a channel to a cognitive user; the bid price is the initial reserve price. On the contrary, $n_{j}>m_{j}$ means demand exceeding supply. When the number of cognitive users oversupply, then the bid price can be determined by linear equations according to the needs of spare channels, that is to say $r_{j}$ increases with the increase of competing user's number.

In the second stage, if $n_{j} \leq m_{j}$, the spare channel is assigned directly to the users whose bid value is greater than the reserve price cognitive users. When $n_{j}>m_{j}$, spectrum owner $j$ will sort the vector portion of the bid value of $B$, and then have all the $m_{j}$ channels assigned to the cognitive users with the highest bid.

On the other hand, as the auction progresses, the numbers of available spectrum and spectrum requirements have undergone changes. Spectrum owners hope there are more participants bid so as to raise the reserve price, and get more economic benefits. For the cognitive users, the judgment of the auction changes over time. In its next round of bids it will draw lessons from the last round of failing bid, and adjust bid strategies, making maximum benefits.

Based on the above analysis we can get an algorithm process based on firstprice sealed auction:

Step 1. Auction begins, and all users (the number is $N$ ) transfer " 1 " bits to spectrum owners

Step 2. Spectrum owners set reserve price $r_{j}$

Step 3. Cognitive users simultaneously submit sealed quotations $b_{j}$

Step 4. Spectrum owners sort $b_{i}$

Step 5. Spectrum owners allocate channels

After the auction, the next round of the auction begins from Step1.

Fig. 2 shows a simple system protocols process, containing three cognitive users (buyers) and two spectrum owners (sellers).

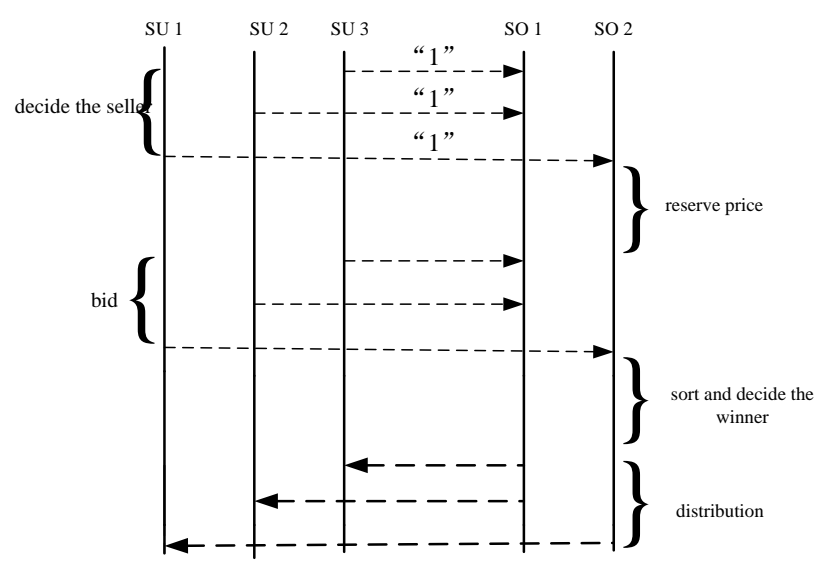

Fig. 2. System protocols process 
The key point of this algorithm is the "layering" process, which is simple and easy to operate. It has the following characteristics:

(1) Low computational complexity. For each spectral owner, in the first stage, it calculates $n_{j}$ times according to the number of " 1 " bits transmitted by cognitive users; in the second stage, it need to sort the bid of $n_{j}$ cognitive users with a stable bubble sorting method, and need to do $n_{j}\left(n_{j}-1\right) / 2$ comparisons. In general, the algorithm has less complexity and higher distribution efficiency.

(2) Low signaling overhead. At each stage of the auction, spectrum owners do not need to get the whole information, each cognitive user only needs to send the corresponding spectrum 1 bit of bid information. Throughout the auction process, cognitive users only need to perceive the idle spectrum around, which does not need to be shared between other cognitive nodes. Each spectrum owner also needn't get all the information of channels and cognitive users.

(3) Stable equilibrium of allocation. Because the cognitive user is rational, the value of each bid is just slightly larger than the reserve price; it has a small chance to get success. It is likely to succeed only when the competition is particularly small and the number of channels available for distribution is bigger than the number of users. During the auction users may switch to other spectrum holders due to the high reserve price, and the system still maximize the overall spectral efficiency.

\subsection{Performance analysis}

\section{A. Nash equilibrium analysis}

Suppose $N$ cognitive users are risk-neutral, the channel value is independent for them, $v_{i j}$ represents the value of a spectrum owner $j$ to cognitive user $i$. Now, we consider the tactic of cognitive User 1. If the value of channel to other SU is higher than the value of cognitive User 1, then in a symmetric equilibrium, participant 1 will not be a winner in the auction. At this time it is unnecessary to consider the optimal bidding strategy. If the cognitive user 1 gets the highest bid price, then the equilibrium strategy is to make its own bid higher than the sub-high value with a small $\varepsilon$.

In addition to cognitive user 1 , there are $N-1$ participating bidders, so the value of channel for the $N-1$ participants is $v$, and the probability of the channel to the second highest value on all participants. Accordingly, $v$ is equal to the expected value

$$
E(v)=\int_{0}^{v_{1 j}} v(N-1)\left(\frac{1}{v_{1 j}}\right)\left(\frac{v}{v_{1 j}}\right)^{N-2} d v=\frac{(N-1) v_{1 j}}{N} .
$$

This is the first price seal auction bidder Nash equilibrium strategy, which is a linear function. Obviously, in the case of Nash equilibrium, cognitive users' bid is lower than the valuation level. When the number of bidders increases, equilibrium bid level will approach the valuation level. In the auction process some cognitive users with little transmitting tasks may transfer to other spectrum owners because the reserve price is too high. However, from the above discussion we can see that the efficiency of spectrum allocation and will not be affected from a global perspective. 


\section{B. Simulation analysis}

We assume that a cognitive user is randomly distributed within a circular area of a radius of about a few KM in a cognitive radio scenario, and the number of channels to be accessed is less than the total number of users for access. According to the above actual scenario, we consider a simple auction situation where the network has only two spectrum owners with 2 spare channels respectively, the total number of cognitive users is $N=16$. We compare the earnings of SO1 under different circumstances with different number of participants.

As is shown in Fig. 3, after 20 rounds of bid in the auction, the revenue is apparently higher when the number of users is large. It is fully consistent with the actual situation: when there is a competition, the auctioneer raises the reserve price, while the bidder will naturally bid as long as the price is within the estimated range, thus maximizing the revenue of the auction. Similarly, when the competition is very severe, 16 users all participate in bidding for the channels of SO1, and then the revenue is near the theoretical maximum. After that, no matter how intense the competition is, the auction revenue will not change a lot. This result is consistent with the theoretical analysis, which further illustrates the effectiveness of the algorithm.

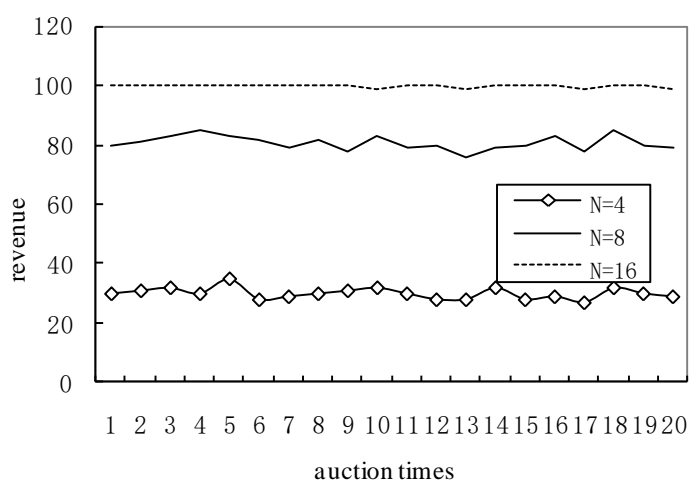

Fig. 3. Revenue of auction

Fig. 4 shows a comparison of the SO1 benefits of the two allocation methods with eight cognitive users who compete for SO1's two idle channels. The results of the analysis were consistent with the expectations. The benefit from the auction of spectrum allocation based on the first price is greater, and auction results are more stable. But for random assignment, the total system revenue does not converge to a relatively stable value, and fluctuations occur.

Finally, we made a comparison of the spectrum efficiency between first-price sealed auction algorithm and greedy algorithm. Fig. 5 shows that when there are more cognitive users to bid, the first-price sealed auction algorithm is able to ensure the stable spectrum utilization and spectrum efficiency in each auction, while the greedy algorithm often has large fluctuations. 


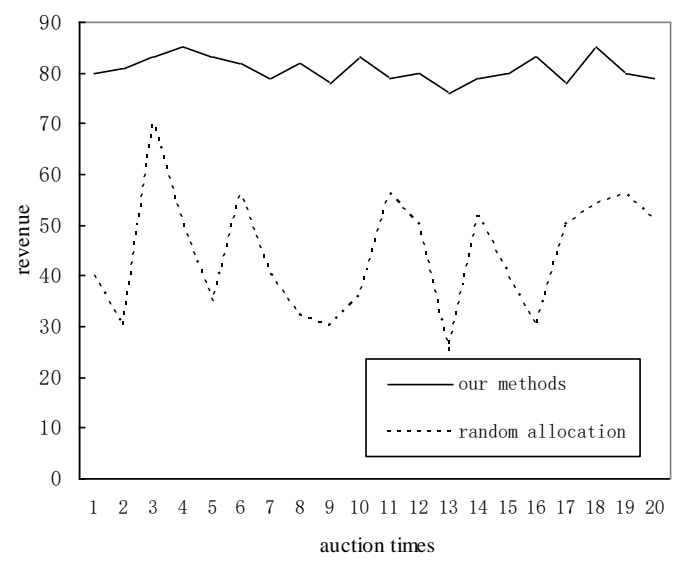

Fig. 4. Comparison of revenue

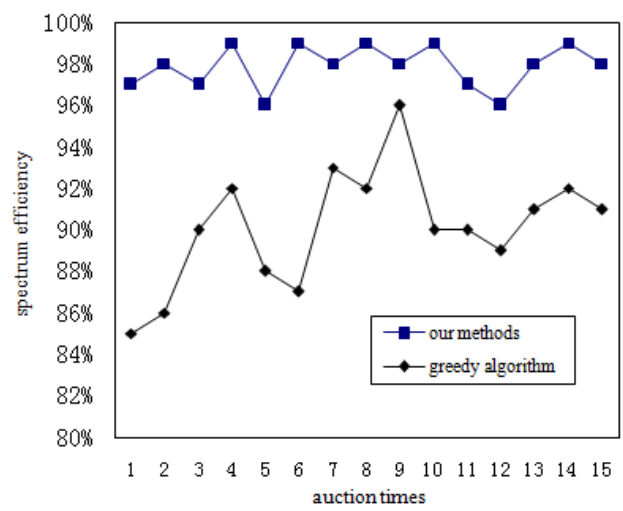

Fig. 5. Comparison of spectrum efficiency

\section{Conclusion}

We analyzed the cognitive radio network system and proposed a spectrum allocation model based on the auction in the network system with a weak central structure. Next, we gave an assumption of an auction model in the cognitive radio system proposing spectrum allocation algorithm based on first-price sealed auction with the existence of multi-carriers. We conducted a performance analysis of the theoretical algorithm, and then operated a simplified network model simulation analysis discussing the revenue of spectrum allocation. It is proved that this algorithm is easy to operate, with low complexity, and can reach a stable equilibrium.

Acknowledgments: This work is supported by the Educational Science Planning Group of Hunan Province (XJK16BYY03) and the Research Foundation of the Education Bureau of Hunan Province (12B115). 


\section{References}

1. B roderso n, R. W., A. Wol is z, D. C a bric et al. CORVUS: A Cognitive Radio Approach for Usage of Virtual Unlicensed Spectrum. http://bwrc.eecs.berkeley.edu/Research/MCMA/CR/Whitepaper_nal1.pdf

2. Mit o l a, J. Cognitive Radio: Making Software Radios More Personal. - IEEE Pers Commun, Vol. 6, 1999, No 4, pp. 13-18.

3. F e n g, W. J., R. J i a n g, P. H a n, W. L i a o, H. H e. Performance Analysis of Cognitive Radio Spectrum Access with Different Primary User Access Schemes. - Wireless Personal Communications, Vol. 75, 2014, No 1, pp. 309-324.

4. Y i, L. G., Y. M. L u. Utility-Driven Relay for Hybrid Access Femtocells Based on Cognitive Radio Spectrum Auction. - Computer Networks, Vol. 80, 2015, No C, pp. 155-166.

5. Li u, Z., C. Li. On Spectrum Allocation in Cognitive Radio Networks: A Double Auction-Based Methodology. - Wireless Networks, 2015, pp. 1-14.

6. J i a n g, W., W. F e n g, Y. Y u. Spectrum Allocation Based on Auction in Overlay Cognitive Radio Network. - Ksii Transactions on Internet \& Information Systems, Vol. 9, 2015, No 9, pp. 3312-3334.

7. Ga ndhi, S., C. B u rag oh a i n, L. C a o et al. A General Framework for Wireless Spectrum Auctions. - In: Proc. of IEEE DySPAN, 2007, No 12, pp. 22-23.

8. Ch e n, B., A. T. Ho an g, Y.-C. Li an g. Cognitive Radio Channel Allocation Using Auction Mechanisms. - VTC Spring, Singapore, 2008, pp. 1564-1568.

9. Ch e n, B., H.-K. W u, A. T. H o a n g et al. Optimizing the Second-Price Auction Algorithm in a Dynamic Cognitive Radio Network. - In: Proc. of 11th IEEE Singapore International Conference Communication Systems (ICCS'2008), 2008, pp. 1514-1518.

10. Niyato, D., E. Hossian. Competitive Pricing for Spectrum Sharing in Cognitive Radio Networks: Dynamic Game, Inefficiency of Nash Equilibrium, and Collusion. - IEEE Journal on Selected Areas in Communications, Vol. 26, 2008, No 1, pp. 192-202.

11. Alsarhan, A., A. Quttoum. Dynamic Auction for Revenue Maximization in Spectrum Market. - Wireless Personal Communications, Vol. 83, 2015, No 2, pp. 1405-1423. 